ENTORNOS, No. 27. | Abril 2014

\title{
Niveles de ruido ambiental en la Universidad Surcolombiana (sede central)
}

\section{Levels of environment noise in the Surcolombiana University (main Campus)}

\section{Niveaus von Umgebungslärm an der Universidad Surcolombiana (hauptsitz)}

\author{
Zully Cuellar ${ }^{1}$, Katherine Diaz ${ }^{2}$, Yolima Taborda ${ }^{3}$
}

\begin{abstract}
Resumen
Esta investigación, realizada por estudiantes del programa de Licenciatura en Ciencias Naturales: Física, Química y Biología, identifica los niveles de ruido ambiental de la Universidad Surcolombiana (sede central), tomando como referencia los estándares permitidos de ruido ambiental en un ente educativo, esto con el objetivo de proponer recomendaciones al sistema de gestión ambiental de la universidad y de esta manera fortalecer el componente de control de ruido ambiental y emisiones atmosféricas por medio de la educación ambiental, instrumento que permitirá la toma de conciencia y participación de la comunidad en la mitigación o prevención de problemas ambientales. Una realidad concreta es el ruido ambiental al cual está sometida la comunidad universitaria el cual se están generando efectos en el campus universitario, entre otros, está la mala comunicación interpersonal, malestar, estrés, que se une a la disminución del rendimiento y la concentración. El estudio de esta problemática ambiental se aborda desde la relación entre individuo, sociedad y naturaleza y de qué tipo de sociedad se quiere.
\end{abstract}

En el marco de un enfoque cuantitativo en todo el plantel educativo se establecieron estratégicamente 18 puntos de toma de datos, en donde se realizaron las mediciones respectivas con un sonómetro, de acuerdo a la Resolución 0627 del 7 de Abril de 2006 del Ministerio de Ambiente, Vivienda y Desarrollo Territorial. Se obtuvo como resultado para los días hábiles - no hábiles en la jornada diurna - nocturna un promedio de $78.4 \mathrm{~dB}$ y $70.5 \mathrm{~dB}$ y $70.6 \mathrm{~dB}$ y $68.8 \mathrm{~dB}$ respectivamente, por lo tanto en la Universidad Surcolombiana (sede central) no se está cumpliendo con los niveles máximos permisibles de ruido ambiental, los cuales son para la jornada diurna $65 \mathrm{~dB}$ y

\begin{abstract}
This research, carry out by students of the degree programme in Natural Sciences: Physics, Chemistry and Biology, identifies the levels of environmental noise of the Colombian South University (headquarters), taking as a reference to the ambient noise standards allowed in an educational entity, this in order to make recommendations to the system of environmental management of the university and thereby strengthen the control component of ambient noise and atmospheric emissions through environmental education, that it will allow the awareness and participation of the community in mitigation or prevention of environmental problems. A concrete reality is the environmental noise which the University community is subjected, which is generating effects on the University campus, and others is the poor interpersonal communication, discomfort, stress, that binds to decrease performance and concentration. The study of this environmental problem is approached from the relationship between individual, society, and nature and what kind of society you want to.
\end{abstract}

Within the framework of a quantitative approach in all the school site were established strategically 18 points of data, where their measurements were made with a sound level meter, according to Resolution 0627 of 7 April 2006 the Ministry of Environment were conducted housing and Territorial Development. The results for working days - non-working days in the day - night was an average of $78.4 \mathrm{~dB}$ and $70.5 \mathrm{~dB}$ and $70.6 \mathrm{~dB}$ and $68.8 \mathrm{~dB}$ respectively, thus at the Surcolombiana University (Headquarters) the maximum permissible ambient noise levels are not being fulfilled, which are for day shifts and night $65 \mathrm{~dB}$ $50 \mathrm{~dB}$ at educational and research centers.

1 - Magister y Especialista en Educación. Universidad Surcolombiana. Neiva. Colombia. zully.cuellar@usco.edu.co

2 - Estudiante del Programa de Licenciatura en Ciencias Naturales: Física, Química y Biología. Universidad Surcolombiana. Neiva. Colombia. jekadito.234@gmail.com

3 - Estudiante del Programa de Licenciatura en Ciencias Naturales: Física, Química y Biología. Universidad Surcolombiana. Neiva. Colombia. yoantava@hotmail.com 
nocturna $50 \mathrm{~dB}$ en centros educativos y de investigación.

Se encontró que hay áreas críticas, sitios con mayor ruido ambiental fueron aquellos en donde se hallaban el tránsito de vehículos, motocicletas, aviones, las entradas y salidas de personal y regiones limítrofes de mucha movilidad de transeúntes como son las avenidas y colegios. Como resultado de la investigación se elaboraron 4 mapas de ruido ambiental ( 2 para días hábiles diurno - nocturno, 2 para días no hábiles diurno - nocturno), los cuales sirvieron como base para formular recomendaciones a la comunidad en general y al sistema de gestión ambiental de la universidad, según la norma nacional del Ministerio de Salud (Resolución 8321 del 1983), e internacionales como el Convenio 148 de 1977 y la Organización Mundial de la Salud.

Palabras clave: Educación, Gestión y Ruido ambiental, Mapas de Ruido.

\section{Zusammenfassung}

Diese Untersuchung, realisiert durch Bachelor-Studenten der Naturwissenschaften Physik, Chemie und Biologie, identifiziert die Niveaus des Umgebungslärms der Universidad Surcolombiana (Hauptsitz) und nimmt als Referenz die erlaubten statistischen Normen von Umgebungslärm an einer Bildungsinstitution, mit dem Ziel, Empfehlungen für das Umweltmanagement der Universität auszusprechen und somit die Kontrollen des Umgebungslärms und der atmosphärischen Emissionen mit Hilfe von Umwelterziehung zu fördern. Dieses Instrument ermöglicht den Einfluss auf das Bewusstsein und die Teilnahme der Gemeinschaft an der Minimierung oder Prävention der Umweltprobleme. Eine konkrete Wirklichkeit des Umgebungslärms, dem die universitäre Gemeinschaft ausgesetzt ist und der sich auf den universitären Campus auswirkt, ist unter anderem eine schlechte interpersonelle Kommunikation, Unwohlsein und Stress, was wiederum das Nachlassen der Leistungsfähigkeit befördert. Das Erforschen dieser Umweltprobleme betrifft die Beziehung zwischen dem Individuum, der Gesellschaft und der Natur und der Art von Gesellschaft, die man sich wünscht.

\section{Introducción}

El ruido se considera como uno de los principales factores de riesgo para la salud humana por los efectos sicológicos, fisiológicos y por su influencia en el normal desarrollo de actividades cotidianas. El ruido al que una persona se ve expuesta depende del ambiente en el que se encuentre. No obstante, los entornos ruidosos más agresivos se producen como consecuencia directa de la actividad humana, por lo tanto, su manifestación más importante tiene lugar en donde se concentran tales actividades.

El ruido como contaminante invisible genera diversos riesgos en la salud, afectando la calidad de vida de la comunidad ya que su producción puede generar efectos crónicos entre estos tenemos según Barbejo (2006): accidentes laborales, afectaciones cardiovasculares, baja productividad, conductas agresivas, malestar/ estrés, pérdida de audición, perdida de atención y desconcentración.

Esta situación se agudiza por la falta de tratamiento como investigación educativa, evidenciado en el diagnóstico de dificultades realizado por el SINA (2002), del Ministerio del Medio Ambiente y del
Ministerio de Educación Nacional, al encontrar pocos resultados en las acciones que ha emprendido la Universidad para incorporar la dimensión ambiental, desde la transversalidad de la temática, en sus procesos de formación, investigación y extensión, (ejes fundamentales de su quehacer).

En este contexto, el desafío es lograr que estas actividades, no repercutan en el estudio y el trabajo de una comunidad, en este caso la comunidad académica de la Universidad Surcolombiana (sede central) ubicada en Neiva departamento del Huila y las personas puedan contribuir a evitar los eventuales daños a que están expuestos diariamente. Para esto el Sistema de Gestión Ambiental (SGA, 2013), se propone reforzar el marco regulatorio y la generación de información, así como también acciones de difusión y educación en el control de ruido ambiental de la universidad.

Para realizar un control eficiente del ruido se debe disponer de información objetiva y cuantificable. En concreto, se debe conocer la situación actual de los niveles de ruido presentados para establecer metas, 
definir y aplicar programas de prevención y control de ruido ambiental, acordes con la realidad de la comunidad universitaria.

Colombia publicó el 7 de Abril del 2006 la Resolución 0627 a través del Ministerio de Ambiente, Vivienda y Desarrollo Territorial; en esta Resolución exige que las ciudades con más de cien mil (100.000) habitantes elaboren mapas de ruido ambiental. De esta manera, como estudiantes de Licenciatura en Ciencias Naturales somos generadores de conciencia ambiental y de procesos que conllevan a la conservación, uso sostenible y manejo de los recursos naturales, reconociendo la responsabilidad legal se plantea la utilización de esta resolución para aplicarla dentro de la Universidad Surcolombiana (sede central) para identificar la contaminación por ruido ambiental, teniendo en cuenta los lineamientos normativos como el horario (diurno - nocturno), días hábiles y no hábiles, mapas de ruido ambiental, etc.

El desconocimiento de problemáticas ambientales por ruido, la escasa formación docente en la dimensión ambiental, la falta de conocimiento que lleva a una ausencia de conciencia sobre éste, hizo que fuera necesario contribuir a resolver esta problemática fortaleciendo la educación ambiental y la gestión ambiental de la universidad con el fin de beneficiar a toda la comunidad universitaria, en este sentido esta propuesta de investigación estudio el fortalecimiento de la educación y gestión ambiental a partir de la identificación de los niveles de ruido ambiental producidos en la Universidad Surcolombiana (Sede central).

Identificamos los niveles de ruido ambiental en el campus universitario en días (hábiles - no hábiles) y en horas (diurno - nocturno) en la Universidad Surcolombiana (sede central), con base a los análisis realizados, partiendo de la norma nacional del Ministerio de Salud (Resolución 8321), e internacional como la Organización Mundial de la Salud (OMS).

\section{Metodología}

Los procedimientos desarrollados para obtener los resultados de la investigación se establecieron teniendo en cuenta la resolución 0627 del 2006 del Ministerio de Ambiente Vivienda y Desarrollo Territorial (MAVDT), la cual reglamenta la toma de datos, elaboración de mapas de ruido ambiental.

En este sentido primero se realizó el recorrido físico de la universidad Surcolombiana delimitando la zona y determinando 18 puntos de medida que se van a monitorear.

Los 18 puntos distribuidos en la universidad fueron:

1. Entrada principal peatonal de la universidad;

2. Pasillo entre ágoras y la cafetería café y letras;

3. Parqueadero vehicular central 1;

4. Parqueadero vehicular de artes;

5. Parqueadero de camionetas de la USCO;
6. Ágoras 2;

7. Parqueadero vehicular central 2;

8. Parqueadero vehicular central 3;

9. Pasillo comunicador con los bloques 18 y 21;

10. Entrada de la cafetería cine café;

11. A un costado del comedor del restaurante;

12. Parqueadero principal de motos;

13. Centro de la cancha de vóley y fútbol playa;

14. Centro del campo de fútbol;

15. Pasillo intercomunicador con el bloque $25,26,27$;

16. Parqueadero vehicular de la facultad de ingeniería;

17. Parqueadero vehicular del bloque 30 ;

18. Entrada del parqueadero vehicular y de motos del bloque 30 .

Según como se aprecia en la figura 1, que representa la ubicación de estos en el mapa de la Universidad Surcolombiana.

Después de la evaluación de la zona y ya teniendo los puntos seleccionados, se establecieron las coordenadas con un GPS y una descripción de lo que rodea el punto para relocalizar cada vez que se vayan hacer lecturas de presión sonora.

Posterior a esto se determinó el número de horas diurnas y nocturnas durante las cuales se efectuaron la toma de mediciones, según la MAVDT (2006, pág. 1 y 2). Por lo tanto cada punto será medido 2 veces en las jornadas diurna y nocturna para un día hábil e igualmente para los días no hábiles, en horarios de medición: DIURNO de las 7:01 a las 21:00 horas y NOCTURNO de las 21:01 a las 7:00 horas.

Con base en esto se establecieron los días en los cuales se efectúa el monitoreo: los puntos seleccionados se distribuyeron en 6 días hábiles (lunes a sábado) y para los días no hábiles se utilizaron los domingos y lunes festivos necesarios hasta completar todo el monitoreo. Se procede entonces a recolectar los datos en los puntos antes señalados, mediante el Informe Técnico de Medición de Ruido Ambiental (Ver anexo A). La instrumentación que se utilizó para efectos de medición de los niveles de presión sonora, para garantizar la fidelidad de los datos, fueron los que propone la norma de emisión de ruido y ruido ambiental: Un sonómetro tipo 2, un anemómetro y un trípode de 4 metros de altura. Además se utilizó: un GPS, computador y una escalera, para complementar la actividad de medición.

Luego viene la tabulación de los datos tomados en cada punto, se identificaron los tipos de ruido y presentaron los resultados consignados en un mapa de ruido ambiental.

Para la elaboración del mapa de ruido ambiental se mantuvieron los contornos de variación de niveles de ruido ambiental, teniendo en cuenta múltiplos de $5 \mathrm{~dB}$ sobre los límites superior e inferior de niveles de Ruido Ambiental, ya que con este se destaca una mayor variabilidad por zonas. Para acciones de desarrollo del 
Figura 1. Mapa de localización de los puntos de medición de ruido ambiental de la Universidad Surcolombiana (sede central). Fuente: http://www.usco.edu.co/pagina/sede-neiva.
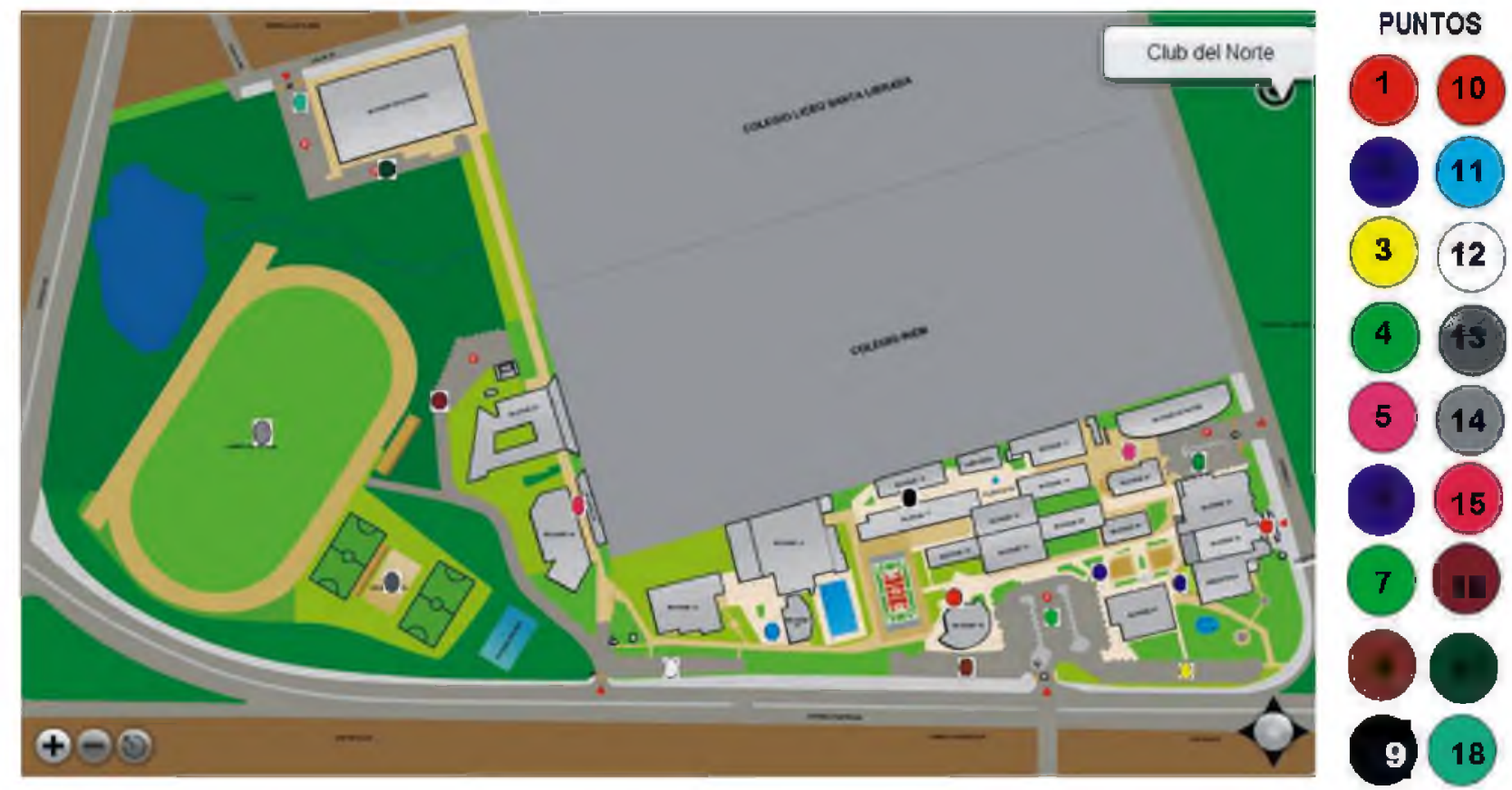

mapa de Ruido Ambiental, se tuvo en cuenta el mapa oficial de la Universidad Surcolombiana (sede central) suministrado por el departamento de planeación en el software autocad, luego determinamos una escala a preferencia y en él se mostró las líneas de nivel (msnm), como los resultados de los niveles de ruido ambiental con sus respectivos colores para representaciones gráficas cada 5 y $10 \mathrm{~dB}(\mathrm{~A})$ como lo indica la norma en su anexo 5.

Paralelo a la realización de los mapas de ruido ambiental, realizamos los análisis de las condiciones atmosféricas (temperatura, velocidad el viento y humedad relativa), con el fin de conocer el comportamiento registrado en los días en que se realiza el monitoreo por presión sonora. La comparación de los resultados con la norma de emisión de ruido y ruido ambiental y los estándares máximos permisibles de niveles de ruido ambiental expresados en decibeles $\mathrm{DB}(\mathrm{A})$ establecidos por la norma de emisión de ruido y ruido ambiental. Para el caso de instituciones educativas y de investigaciones es de $65 \mathrm{~dB}$ en la jornada diurna y $50 \mathrm{~dB}$ en la jornada nocturna.

Este análisis y los mapas de ruido generados, permitió la identificación de las áreas críticas, vulnerables y no vulnerables para el día - noche, detectando las partes más afectadas y menos afectadas por el ruido ambiental.

Finalmente escribimos en un documento oficial, recomendaciones con base a los análisis realizados y áreas críticas encontrados, partiendo de la norma nacional del Ministerio de Salud (Resolución 8321), e internacional como la Organización Mundial de la Salud (OMS). Se entregó al sistema de gestión ambiental de la Universidad Surcolombiana las recomendaciones, entregaron volantes a la comunidad sobre los resultados de la investigación y en diferentes zonas de la universidad se instalaron 4 retablos que contienen los mapas de ruido ambiental producto de la investigación.

\section{Resultados y Discusión}

Después de obtener los resultados de presión sonora provenientes del informe técnico de medición de ruido ambiental y realizar los cálculos para obtener los datos de presión sonora equivalente, resultante con sus respectivos ajustes en la jornada diurna y nocturna para los días hábiles y no hábiles, se procedió a consignarlos en el mapa de ruido ambiental representado en gamas de colores.

Para obtener un análisis del conjunto de datos recolectados en decibeles se acude a la Resolución 0627 del 2006, donde en el anexo 5, describe cómo los resultados de los niveles de ruido obtenidos en $\mathrm{dB}(\mathrm{A})$ equivalentes, se pueden usar para la representación gráfica, por cada $5 \mathrm{~dB}(\mathrm{~A})$, desde 35 a $85 \mathrm{~dB}(\mathrm{~A})$. Se reconocieron los niveles de ruido en $\mathrm{dB}(\mathrm{A})$ en un conglomerado total (Mapa de Ruido Ambiental), ofreciendo una descripción de los datos obtenidos de forma numérica y visual. Antes se organizaron los resultados según los colores asignados para cada dato, como se puede observar en la tabla 1 para días hábiles y no hábiles. Se agregó el color azul más oscuro que representa $85 \mathrm{~dB}$ en adelante como lo indica el protocolo de medición de ruido ambiental. 
Tabla 1. Combinación de colores para representaciones graficas (mapas de ruido ambiental) en los días hábiles y no hábiles, jornada diurna - nocturna.

\begin{tabular}{|c|c|c|c|c|c|c|c|c|}
\hline \multicolumn{5}{|c|}{ Días Hábiles } & \multicolumn{4}{|c|}{ Dias No Hábiles } \\
\hline Punto & $\begin{array}{l}L_{\text {min }}(d B) \\
\text { Diurno }\end{array}$ & Color & $\begin{array}{c}L_{\text {Nat }}(d B) \\
\text { Hocturno }\end{array}$ & Color & $\begin{array}{l}L_{\text {ani }} \text { (dB) } \\
\text { Diurno }\end{array}$ & Colar & $\begin{array}{c}L_{\text {sa }}(d B) \\
\text { Nacturno }\end{array}$ & Color \\
\hline 1 & 84.7 & & 76.3 & & 75.1 & & 76.6 & \\
\hline 2 & 75.3 & & 61.2 & & 75.8 & & 64.6 & \\
\hline 3 & 94.0 & & 65.0 & & 77.9 & & 66.7 & \\
\hline 4 & 84.6 & & 71.5 & & 75.6 & & 56.0 & \\
\hline 5 & 75.8 & & 69.1 & & 64.9 & & 74.8 & \\
\hline 6 & 76.9 & & 73.8 & & 69.1 & & 69.3 & \\
\hline 7 & 92.9 & & 69.0 & & 72.9 & & 77.8 & \\
\hline 8 & 81.2 & & 69.2 & & 75.4 & & 73.0 & \\
\hline 9 & 77.7 & & 73.2 & & 66.4 & & 71.6 & \\
\hline 10 & 74.5 & & 67.6 & & 63.4 & & 58.6 & \\
\hline 11 & 82.6 & & 76.1 & & 68.1 & & 66.4 & \\
\hline 12 & 82.7 & & 75.2 & & 66.0 & & 69.5 & \\
\hline 13 & 72.2 & & 75.1 & & 69.9 & & 83.5 & \\
\hline 14 & 73.5 & & 73.6 & & 86.4 & & 83.2 & \\
\hline 15 & 74.5 & & 64.2 & & 58.7 & & 65.1 & \\
\hline 16 & 66.9 & & 62.4 & & 62.5 & & 58.7 & \\
\hline 17 & 73.7 & & 65.1 & & 67.2 & & 60.2 & \\
\hline 18 & 66.9 & & 81.8 & & 61.9 & & 62.1 & \\
\hline
\end{tabular}

Fuente: Resolución 0627 de 2006 y los autores

Los cuatro mapas de ruido ambiental que se generaron para la Universidad Surcolombiana (sede central), a partir de los resultados fueron en la franja horaria diurna -nocturna en días hábiles; y diurna -nocturna en días no hábiles.

En las figuras 1 y 2 podemos apreciar los mapas de ruido ambiental para los días hábiles (horario diurno) y no hábiles (horario nocturno) respectivamente.

Figura 1. Mapa de Ruido Ambiental de la Universidad Surcolombiana (sede central) de la Franja Horaria diurna dias hábiles.

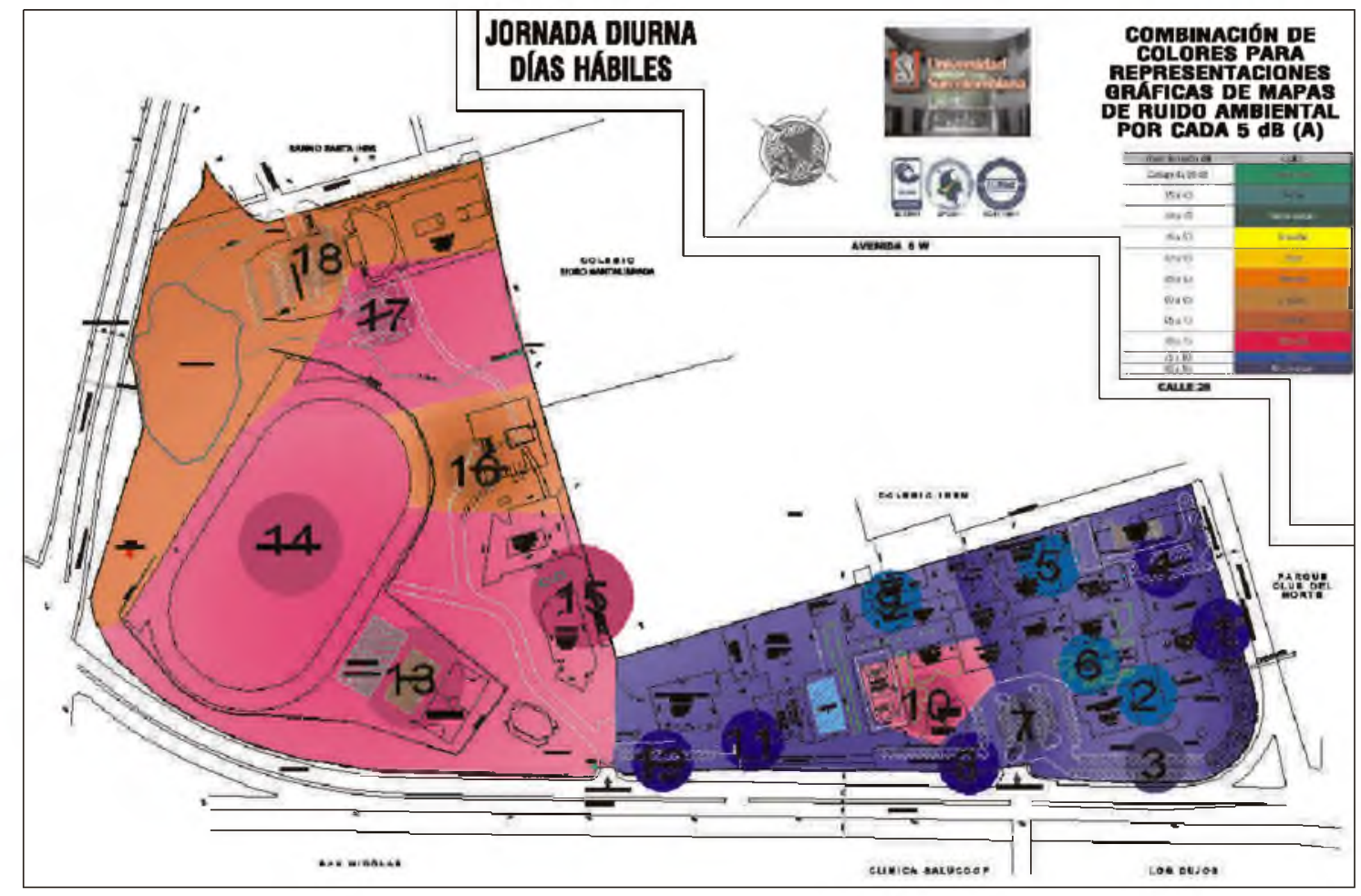


Figura 2. Mapa de Ruido Ambiental de la Universidad Surcolombiana (Sede Central) de la Franja Horaria Nocturna - días no hábiles.

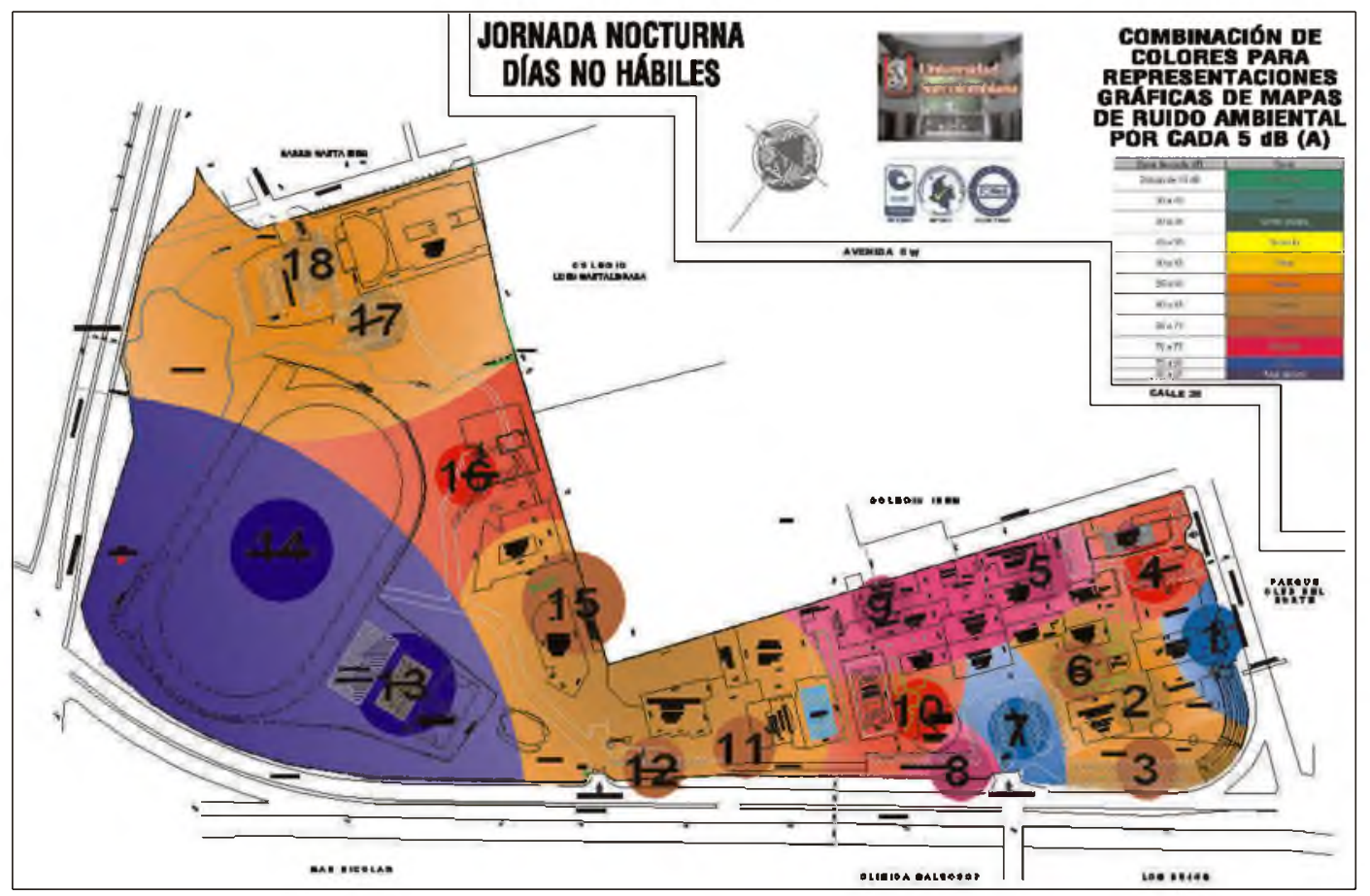

Según la Resolución 0627 (2006), los mapas de ruido son utilizados como documento básico para conocer la realidad de ruido ambiental en la población y poder desarrollar planes, programas y proyectos preventivos, correctivos o de seguimiento. Estos sirvieron para ubicar las zonas críticas y vulnerables. Como se puede apreciar lo colores (gama de azules, rojos y fucsias) en los mapas de ruido están indicando que superamos los niveles requeridos por la norma.

Los resultados arrojados se compararon con los que indica la norma que son de $65 \mathrm{~dB}$ en la jornada diurna y $50 \mathrm{~dB}$ en la jornada nocturna para centros educativos y de investigación.

Para los días hábiles, jornada diurna - nocturna se dieron los siguientes resultados ajustados de presión sonora, los cuales se compararon con los referentes de la Resolución 0627 de 2006. Como se puede apreciar en la tabla 2, no se cumple con la norma pues los $\mathrm{dB}$ pasan de 65 en el diurno y de $50 \mathrm{~dB}$ en el nocturno.

Tabla 2. Resultados ajustados de presión sonora comparados con los referentes de la Resolución 0627 de 2006. (Días hábiles).

\begin{tabular}{|c|c|c|c|c|c|c|}
\hline $\begin{array}{l}\text { Puntos de } \\
\text { monitoreo }\end{array}$ & $\begin{array}{l}\text { LAoq (dB) } \\
\text { Dlurno }\end{array}$ & $\begin{array}{l}\text { Lavo (dB) } \\
\text { norma } \\
\text { Dlumo }\end{array}$ & $\begin{array}{l}\text { ¿Cumple } \\
\text { con la } \\
\text { norma? }\end{array}$ & $\begin{array}{l}\text { LAoq (dB) } \\
\text { Nocturno }\end{array}$ & $\begin{array}{l}\text { Lada (dB) } \\
\text { norma } \\
\text { Nocturno }\end{array}$ & $\begin{array}{l}\text { ¿Cumple } \\
\text { con la } \\
\text { norma? }\end{array}$ \\
\hline 1 & 84.7 & 65.0 & NO & 76.3 & 50.0 & NO \\
\hline 2 & 75.3 & 65.0 & NO & 61.2 & 50.0 & NO \\
\hline$\overline{3}$ & 94.0 & 65.0 & NO & 65.0 & 50.0 & NO \\
\hline 4 & 84.6 & 65.0 & NO & 71.5 & 50.0 & NO \\
\hline 5 & 75.8 & 65.0 & NO & 69.1 & 50.0 & NO \\
\hline 6 & 76.9 & 65.0 & NO & 73.8 & 50.0 & NO \\
\hline 7 & 92.9 & 65.0 & No & 69.0 & 50.0 & NO \\
\hline 8 & 81.2 & 65.0 & NO & 69.2 & 50.0 & NO \\
\hline 8 & 77.7 & 65.0 & NO & 73.2 & 50.0 & NO \\
\hline 10 & 74.5 & 65.0 & NO & 67.6 & 50.0 & NO \\
\hline 11 & 82.6 & 65.0 & NO & 76.1 & 50.0 & NO \\
\hline 12 & 82.7 & 65.0 & No & 75.2 & 50.0 & NO \\
\hline 13 & 72.2 & 65.0 & NO & 75.1 & 50.0 & NO \\
\hline 14 & 73.5 & 65.0 & NO & 73.6 & 50.0 & NO \\
\hline 15 & 74.5 & 65.0 & NO & 64.2 & 50.0 & NO \\
\hline 16 & 66.9 & 65.0 & NO & 62.4 & 50.0 & NO \\
\hline 17 & 73.7 & 65.0 & NO & 65.1 & 50.0 & NO \\
\hline 18 & 66.9 & 65.0 & NO & 81.8 & 50.0 & NO \\
\hline
\end{tabular}


Resumiendo, la figura 3 nos muestra los resultados de niveles de presión sonora en días hábiles, jornada diurna-nocturna.

Figura 3. Resultados ajustados de presión sonora comparados con los referentes de la Resolución 0627 de 2006. (Días hábiles, jornada diurna - nocturna).

\section{COMPARACIÓN CON LA TABLA 2 DE LA RESOLUCIÓN 0627 DE 2006}

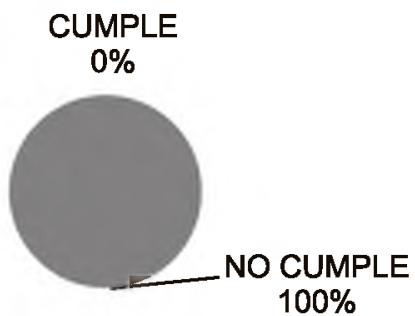

Los resultados arrojados indican que:

En la Jornada Diurna hay áreas críticas porque al comparar los resultados con la Resolución 0627 de 2006 , los niveles de presión sonora se mantuvieron en un rango de $66.9 \mathrm{~dB}$ a $94.0 \mathrm{~dB}$, por lo tanto se halló que el $100 \%$ de los puntos de monitoreo no cumplen con la norma, estos superan los máximos permisibles entre 1.9 a $29.0 \mathrm{~dB}$. Estas fueron: la entrada principal peatonal de la universidad, parqueadero vehicular central 1, parqueadero vehicular de artes, parqueadero vehicular central 2 y el parqueadero vehicular central 3 , son los que se encontraron como área critica, su causa se debe el estar cerca de la carrera $1^{\text {a }}$, calle 26 , aeropuerto, circulación de personas, aires acondicionados encendidos, televisores, equipos de sonido, etc.

Para la Jornada Nocturna se presenta una situación similar, donde se mantuvieron rangos entre 61.2 a 81.8 $\mathrm{dB}$, por lo tanto se determinó que el $100 \%$ de los puntos valorados no cumplen con los estándares permisibles de presión sonora, estos superan entre 11.2 a $31.8 \mathrm{~dB}$ a lo estipulado en la norma. Los puntos que fueron: la entrada principal peatonal de la universidad, parqueadero vehicular de artes, ágoras 2 , a un costado del comedor del restaurante, parqueadero principal de motos, centro de la cancha de vóley y fútbol playa, centro del campo de fútbol y entrada del parqueadero vehicular y de motos del bloque 30, estas son las áreas críticas donde el mayor contaminador es la comunidad universitaria, además porque en la parte sur - occidental se encuentran los bares y discotecas y las vías aledañas a la universidad.

En cuanto a los resultados obtenidos para los días no hábiles, jornada diurna-nocturna, se compararon con la tabla 2 de la Resolución 0627 de 2006 y encontramos que en la mayoría de los puntos tampoco se cumple la norma, pues se encuentran niveles superiores a $65 \mathrm{~dB}$ para el diurno y $50 \mathrm{~dB}$ para el nocturno, como se puede apreciar en la tabla 3.

Tabla 3. Resultados ajustados de presión sonora comparados con los referentes de la Resolución 0627 de 2006. (Días no hábiles).

\begin{tabular}{|c|c|c|c|c|c|c|}
\hline \multicolumn{7}{|c|}{ COMPARACIÓN CON LA TABLA 2 DE LA RESOLUCIÓN 0627 DE 2006} \\
\hline $\begin{array}{l}\text { Puntos de } \\
\text { monitoreo }\end{array}$ & $\begin{array}{l}L_{\text {Aeq }} \\
\text { (dB) } \\
\text { Diurno }\end{array}$ & $\begin{array}{l}\text { LAeq } \\
\text { (dB) } \\
\text { norma } \\
\text { Diurno }\end{array}$ & $\begin{array}{l}\text { ¿Cumple } \\
\text { con la } \\
\text { norma? }\end{array}$ & $\begin{array}{l}\mathrm{L}_{\text {Aeq }}(\mathrm{dB}) \\
\text { Nocturno }\end{array}$ & $\begin{array}{l}\mathrm{L}_{\text {Aeq }}(\mathrm{dB}) \\
\text { norma } \\
\text { Nocturno }\end{array}$ & $\begin{array}{l}\text { ¿Cumple } \\
\text { con la } \\
\text { norma? }\end{array}$ \\
\hline 1 & 75.1 & 65.0 & NO & 76.6 & 50.0 & NO \\
\hline 2 & 75.8 & 65.0 & NO & 64.6 & 50.0 & NO \\
\hline 3 & 77.9 & 65.0 & NO & 66.7 & 50.0 & NO \\
\hline 4 & 75.6 & 65.0 & NO & 56.0 & 50.0 & NO \\
\hline 5 & 64.9 & 65.0 & SI & 74.8 & 50.0 & NO \\
\hline 6 & 69.1 & 65.0 & NO & 69.3 & 50.0 & NO \\
\hline 7 & 72.9 & 65.0 & NO & 77.8 & 50.0 & NO \\
\hline 8 & 75.4 & 65.0 & NO & 73.0 & 50.0 & NO \\
\hline 9 & 66.4 & 65.0 & NO & 71.6 & 50.0 & NO \\
\hline 10 & 63.4 & 65.0 & $\mathrm{Sl}$ & 58.6 & 50.0 & NO \\
\hline 11 & 68.1 & 65.0 & NO & 66.4 & 50.0 & NO \\
\hline 12 & 66.0 & 65.0 & NO & 69.5 & 50.0 & NO \\
\hline 13 & 69.9 & 65.0 & NO & 83.5 & 50.0 & NO \\
\hline 14 & 86.4 & 65.0 & $\mathrm{NO}$ & 83.2 & 50.0 & $\mathrm{NO}$ \\
\hline 15 & 58.7 & 65.0 & SI & 65.1 & 50.0 & NO \\
\hline 16 & 62.5 & 65.0 & SI & 58.7 & 50.0 & NO \\
\hline 17 & 67.2 & 65.0 & NO & 60.2 & 50.0 & NO \\
\hline 18 & 61.9 & 65.0 & SI & 62.1 & 50.0 & NO \\
\hline
\end{tabular}


Resumiendo las imágenes 4 nos muestra los resultados en días no hábiles, jornada diurnanocturna:

Imágenes 4. Resultados ajustados de presión sonora comparados con los referentes de la Resolución 0627 de 2006. (Días no hábiles, jornada diurna-nocturna).

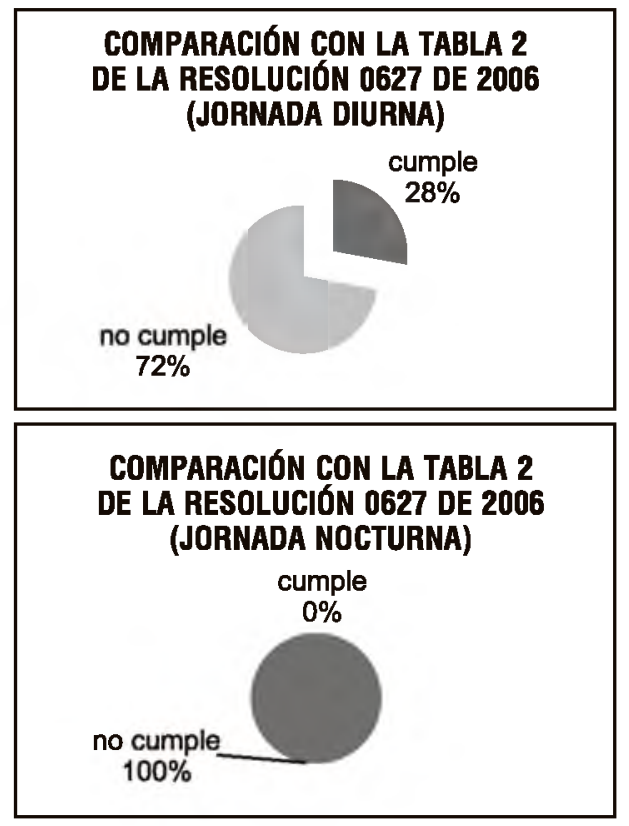

Estos resultados nos indican que:

En la Jornada Diurna los niveles de presión sonora para la Universidad Surcolombiana (sede central) en la jornada diurna se mantuvieron en un rango de $58.7 \mathrm{~dB}$ a $86.4 \mathrm{~dB}$, donde el $27.8 \%$ de los puntos monitoreados cumplen con la norma, por el contrario el $72.2 \%$ de estos supera los máximos permisibles entre $1 \mathrm{~dB}$ a $21.4 \mathrm{~dB}$. Los puntos que presentaron mayor elevación de presión sonora fueron: la entrada principal peatonal de la universidad, pasillo entre ágoras y la cafetería café y letras, parqueadero vehicular central 1, parqueadero vehicular de artes, parqueadero vehicular central 3 y centro del campo de fútbol. Se encontraron áreas críticas de contaminación por ruido ambiental enmarcadas entre los rangos de 72.9 $\mathrm{dB}$ a $86.4 \mathrm{~dB}$, que corresponde a los puntos $1,2,3,4,7$, 8 y 14. Es claro que para estas áreas existe 1 factor determinante que influye en la generación de ruido, está dado por el alto tráfico vehicular.

En la Jornada Nocturna se evidencia que el 100\% de los puntos monitoreados de la jornada nocturna no cumplen con la norma de emisión de ruido y ruido ambiental, manteniendo un rango de $56.0 \mathrm{~dB}$ a $83.5 \mathrm{~dB}$, estos niveles se superaron entre $6 \mathrm{~dB}$ a $33.5 \mathrm{~dB}$. Los puntos que presentaron mayor elevación de presión sonora son: la entrada principal peatonal de la universidad, parqueadero de camionetas de la USCO, parqueadero vehicular central 2, centro de la cancha de vóley y fútbol playa y centro del campo de fútbol.
Encontramos áreas críticas de contaminación por ruido ambiental para la jornada nocturna enmarcados entre los rangos de $60.2 \mathrm{~dB}$ a $83.5 \mathrm{~dB}$, que corresponde a los puntos $1,2,3,5,6,7,8,9,11,12,13,14,15,17 \mathrm{y}$ 18. Es claro que para estas áreas existen dos factores determinantes que influyen en la generación de ruido, el primero: la alta acumulación de establecimientos comerciales (bares, discotecas, etc.), los cuales utilizan sistema de sonido en áreas de espacio público y no cuentan con sistemas de insonorización que garanticen que los niveles de ruidos generados no traspasarán los límites de la propiedad del establecimiento que está generando el ruido y el segundo está dado por el alto tráfico vehicular.

Estos análisis nos permiten dar a la comunidad universitaria recomendaciones para proteger su salud física y mental como:

Receta Para un Día Tranquilo: Respeta los derechos de los demás. (Durante todo el día).

$\checkmark \quad$ Presta atención a los ruidos que hace y respeta el derecho de la comunidad a tener paz, tranquilidad, intimidad y descanso.

$\checkmark$ Baja el volumen del equipo de sonido, radio, televisión, celular, Tablet, lap-top y MP4. Cuando decida escuchar música, ver la televisión, hablar por celular o charlar, asegúrese de que sólo lo escuchan usted y su compañía.

$\checkmark \quad$ No utilice el claxon (pito) de su vehículo salvo en caso de inminente peligro.

$\checkmark$ No practique conductas ruidosas: gritos, portazos, taconeos u otros objetos.

$\checkmark \quad$ Al salir de la oficina y aulas de clase asegúrese de apagar el aire acondicionado.

$\checkmark \quad$ En caso que decida permanecer en un sitio de alto nivel de contaminación de ruido (Ágoras, Cafetería, y Hall) utilice el protector personal auditivo (tapón en espiral).

$\checkmark$ Encienda la motocicleta afuera de las instalaciones de la Universidad.

$\checkmark \quad$ En los momentos de diversión, recuerda que el respeto a los demás es la base imprescindible para una buena convivencia.

$\checkmark$ Aunque sea parte de su familia, no permita que su mascota perjudique el derecho de las personas al descanso, la intimidad y tranquilidad.

$\checkmark$ Aprenda a disfrutar del silencio y la tranquilidad.

Haz respetar tus derechos:

$\checkmark \quad$ Infórmese de sus derechos en relación al ruido. Exija el cumplimento sin reparo sobre el decreto 948 de 1995.

$\checkmark$ Solicite que bajen el volumen de la música y televisor, cuando lo consideres elevada en los lugares como: Ágoras, pasillos, cafetería, gimnasio, y hall.

$\checkmark$ Apóyese del Sistema de Gestión Ambiental para que su acción sea más eficaz.

Contribuye a crear una opinión pública informada: Infórmese y corre la voz sobre los riesgos del culto al 
ruido (droga acústica), el uso excesivo de auriculares, motocicletas, equipos. Transmítaselo a sus hijos, parientes, amigos o conocidos.

\section{Conclusiones}

Acudiendo a la Resolución 0627 del 2006 se obtuvo como resultado para los días hábiles - no hábiles en la jornada diurna - nocturna un promedio de $78.4 \mathrm{~dB}$ y $70.5 \mathrm{~dB}$ y $70.6 \mathrm{~dB}$ y $68.8 \mathrm{~dB}$ respectivamente, por lo tanto en la Universidad Surcolombiana (Sede Central) no se está cumpliendo con los niveles máximos permisibles de ruido ambiental los cuales son para la jornada diurna $65 \mathrm{~dB}$ y nocturna $50 \mathrm{~dB}$ en centros educativos y de investigación. Para los días hábiles - no hábiles en las jornadas diurnas - nocturnas se encontró que en la Universidad Surcolombiana (Sede Central) hay áreas críticas lo cual indica que supera los niveles máximos permisibles de ruido ambiental.

A partir de los análisis realizados podemos encontrar áreas vulnerables los días hábiles - jornada diurna en los puntos 16 y 18 pues solo se halla un sobre paso de $1.9 \mathrm{~dB}$ a lo que indica la norma. Para los días hábiles jornada nocturna, no se hallaron áreas vulnerables debido a que todos los puntos monitoreados se encuentran en áreas críticas.

En los días no hábiles - jornada diurna, las áreas vulnerables por contaminación de ruido ambiental están enmarcados entre los rangos de $66.0 \mathrm{~dB}$ a 69.9 $\mathrm{dB}$, que corresponde a los puntos $6,9,11,12,13$ y 17 . Existen 2 factores determinantes que influyen en la generación de ruido, el primero por los aires acondicionados que no dejan apagados en algunas oficinas administrativas y el segundo el tráfico rodado. Para la jornada nocturna, las áreas vulnerables están enmarcados entre los rangos de $56.0 \mathrm{~dB}$ a $58.7 \mathrm{~dB}$, que corresponde a los puntos 4,10 y 16 . Pues solo se halla un sobre paso de $6 \mathrm{~dB}$ a $8 \mathrm{~dB}$ a lo que indica la norma, que con unas recomendaciones básicas se puede bajar el nivel de presión sonora.

A partir de los análisis se originaron 4 mapas de ruido: Franja Horaria diurna - días hábiles, Franja Horaria Nocturna - días hábiles, Franja Horaria diurna - días no hábiles y Franja Horaria Nocturna - días no hábiles. Estos permitieron la generación de recomendaciones a la comunidad universitaria y al sistema de gestión ambiental para fortalecer la educación ambiental, haciendo que el individuo se apropie de la realidad concreta y generar en él y en la comunidad actitudes de valoración y respeto por el ambiente.

Se encontró que a nivel interno de la universidad el mayor contaminante por ruido ambiental era producido por gritos, charlas fuertes, televisores, reproductores de música, aires acondicionados, entre otros objetos. A nivel externo las fuentes principales fueron el paso de vehículos pesados (tracto mulas, volquetas, carro tanques y camiones), automóviles, motocicletas, bares, discotecas, restaurantes, clínica y colegios que rodean a la Universidad Surcolombiana (Sede Central) aumentando los niveles máximos permisibles de ruido ambiental.

A partir de la experiencia se obtuvo el desarrollo de competencias conceptuales, procedimentales y actitudinales para la enseñanza - aprendizaje de las ciencias y educación ambiental en los autores que pertenecen a la Licenciatura en Educación Básica con Énfasis en Ciencias Naturales y Educación Ambiental en los componentes que incluye un estudio de ruido ambiental (manejo de normas nacionales e internacionales, equipos de medición, ejecución de un mapa de ruido y análisis de acuerdo a la Resolución 0627 de 2006).

Se realizaron recomendaciones como aporte para que el sistema de gestión ambiental organice un plan de mitigación de ruido y a través de éste se fortalezca la educación ambiental. Estas recomendaciones recogen los principios que toda educación ambiental debe tener según SINA, (2012) como son la formación de individuos en la toma de decisiones responsables del desarrollo sostenible, la facilitación de la comprensión de la naturaleza compleja del ambiente, la identificación de los problemas ambientales del entorno para tener herramientas de reflexión frente a este. Para que esto sea factible le corresponde al Sistema de Gestión Ambiental llevarlas a cabo por medio de plan de mitigación que permitan proporcionar un ambiente armónico y de calidad de vida para la comunidad universitaria, con base a los análisis realizados y áreas críticas encontradas desde el foco hasta el receptor.

Esta investigación da la posibilidad de realizar próximas investigaciones en emisión de ruido, efectos en la salud por ruido ambiental, contaminación acústica.

\section{Referencias Bibliográficas}

Barbejo, J. (2006). Ruido: El Enemigo Invisible. Revista de Lex nova. Pág. 5.

La Organización Internacional De Normalización. (1996). Acústica Descripción y Medición del Ruido Ambiental. España.

Política Nacional De Educación Ambiental (SINA). (2002). Ministerio del Medio Ambiente. Ministerio de Educación Nacional. Bogotá, DC. Pág. 12, 18, 19, 20, 21, 22, 39 .

Resolución 0627 De 2006. Ministerio de Ambiente, Vivienda Y Desarrollo Territorial. Bogotá-Colombia, págs. 2, 3, 6, 7, 8, 9, 20 , $28,29$.

Resolución 8321 De 1983. Ministerio de Salud. Normas sobre la Protección y Conservación de la Audición de la Salud y el Bienestar de las personas, por causa de la protección y emisión de Ruidos. Bogotá-Colombia, pág.9-10.

Sistema De Gestión Ambiental. (Última actualización 2013, mayo 17). Universidad Surcolombiana. Consulta realizada el 10 de mayo de 2013, en http://www.usco.edu.co/pagina/sga

The Noise Control Center (USA) Consulta realizada en Mayo 15 de 2013, en http://www.cdc.gov/niosh/topics/noise/prevention.html. 


\section{Anexos}

Anexo A: Informe Técnico de Medición de Ruido Ambiental en la Universidad Surcolombiana (Sede Central). Información General

\begin{tabular}{|l|l|l|}
\hline Responsables del & Ubicación de la medición: & Fecha de medición:
\end{tabular} informe:

Latitud: $\quad$ Longitud:

\begin{tabular}{|l|l|l|}
\hline Número de punto: & Hora inicial de medición: & Hora final de medición:
\end{tabular}

Equipos utilizados

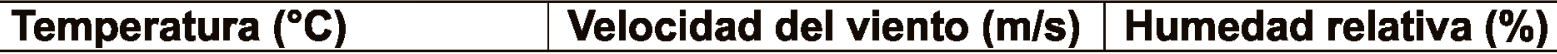

Características de la medición

\begin{tabular}{|l|l|l|l|}
\hline Dirección del & Lmín (dB) & Lmáx (dB) & Leq (dB)
\end{tabular}

punto de medición

Norte

Sur

Oriente

Occidente

Vertical hacia arriba

Automóviles Motos les

Buses de servicio público
Camionetas

Aviones

Tractomulas Camiones

Observaciones: 